\section{A patient with anaphylaxis to diphenhydramine without cross-reactivity to loratadine}

Elaine Y.L. Ay, MBBS, FHKAM (Medicine), FRCPA, FHKAM (Pathology), D (ABMLI) $)^{a, b}$,

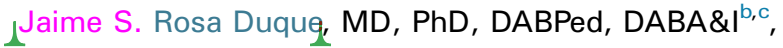
$\Lambda^{C h a k}$ Sing Lay, MBChB, MD (Hons), FRCP (Edin, Glasg, Lond), FHKCP, FHKAM (Medicine) ${ }^{\mathrm{b}}$, and

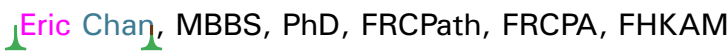
$(\text { Pathology })^{a}$

\section{Clinical Implications}

- Anaphylaxis to antihistamines is rare. In this case, the causative antihistamine was confirmed by repeated clinical reactions shortly after every exposure, as well as skin test and basophil activation tests, which were also helpful for identifying an alternative for the patient.

\section{TO THE EDITOR:}

Antihistamines are common over-the-counter medications used to alleviate symptoms of allergies, insomnia, nausea, and symptoms of viral illnesses. It is exceedingly rare that these drugs cause IgE-mediated, type I reactions. Previously, there were 2 reported cases of anaphylaxis to diphenhydramine. Mur Gimeno et $\mathrm{al}^{1}$ reported a patient with suspected diphenhydramineinduced anaphylaxis and negative skin test, who developed recurrent anaphylaxis on challenge with diphenhydramine. Nevertheless, she tolerated loratadine. Barranco et $\mathrm{al}^{2}$ reported another case of diphenhydramine anaphylaxis in a nonatopic patient who had positive intradermal skin test and challenge but negative prick test and specific IgE. This patient subsequently tolerated terfenadine, cetirizine, loratadine, and dexchlorpheniramine. Here we present the third case of anaphylaxis to diphenhydramine. This is the first case to be reported that involves the use of basophil activation test (BAT) in the diagnosis and management.

A 42-year-old generally healthy man was referred for assessment of recurrent anaphylaxis after taking several medications to alleviate symptoms due to upper respiratory viral illnesses. Over the course of many years, he experienced several episodes of generalized, pruritic, urticarial eruption after using these medications. He became more concerned when, 3 years before his visit to our allergy clinic, he suffered a severe reaction that included diffuse urticaria, flushing, and angioedema, coupled with shortness of breath and dizziness within 5 minutes after the application of the over-the-counter intranasal drops, Coldistan. His anaphylactic response was so intense that he was transferred to the intensive care unit and required an adrenaline infusion. Review of the packaging label of Coldistan revealed that the active ingredients consisted of diphenhydramine and naphazoline with no excipients listed. Serum tryptase during the event was

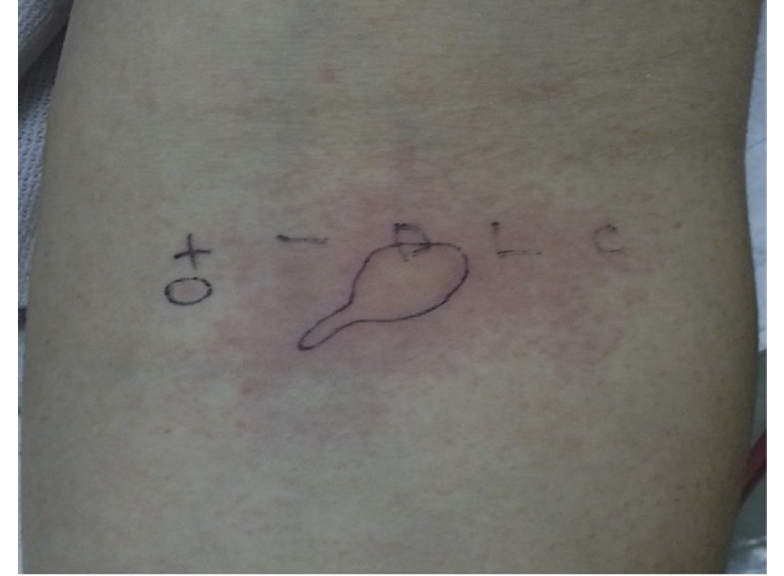

17172185 Skin Test read at $20 \mathrm{~min}$

Skin Prick Test

Positive Negative Diphenhydramine Loratadine Cetirizine (Histamine) (50\% Gly (50 mg/ml) (5 mg/ml) (10 mg/ml) $50 \%$ COCAS)

FIGURE 1. Skin prick test results at 20 minutes.

significantly elevated compared with baseline (57.9 and $6.2 \mu \mathrm{g} / \mathrm{L}$, respectively), supporting the diagnosis of anaphylaxis. Two years later, a similar reaction recurred after he took diphenhydramine orally for an upper respiratory illness. This time, he was stabilized after treatments for anaphylaxis in the emergency unit.

Skin prick tests with diphenhydramine $(50 \mathrm{mg} / \mathrm{mL})$, cetirizine $(10 \mathrm{mg} / \mathrm{mL})$, and loratadine $(10 \mathrm{mg} / \mathrm{mL})$ were performed. ${ }^{1}$ Epicutaneous prick with preservative-free diphenhydramine yielded positive skin reactivity (wheal diameters: histamine $6 \times 6 \mathrm{~mm}$, saline $0 \mathrm{~mm}$, diphenhydramine $13 \times 19 \mathrm{~mm}$ with pseudopod), whereas loratadine and cetirizine prick results were negative (Figure 1). Diphenhydramine prick tests were negative in 6 healthy subjects. We performed BAT with serial dilution of the drugs from neat up to 10,000 fold (BAT reagents from Flow CAST BÜHLMANN Laboratories AG, Schönenbuch, Switzerland). The intravenous formulation of diphenhydramine (Omega Laboratori as used. The results showed positive reactivity for diphen $\&$ mine (Figure 2) and cetirizine but were negative for chlorpheniramine and loratadine. BATs for 4 healthy control subjects were tested negative for all 4 antihistamines. Based on these results, the patient agreed to an open challenge to loratadine, which he tolerated.

This patient's presentation was most consistent with an IgEmediated process as evident by reproducibility of symptoms on self-challenge, an elevated serum tryptase level after exposure, and positive skin reactivity and BAT results. ${ }^{3,4}$ Anaphylaxis after intranasal administration of a drug is rare, but it is possible. A patient had even suffered cardiac arrest due to anaphylaxis after applying intranasal chlorhexidine. ${ }^{5}$ Diphenhydramine is a low molecular weight compound, and as such the underlying mechanism leading to reaction is likely a hapten effect caused by the drug itself or its metabolite(s). In the 2 previous reported cases, one patient had a negative skin test result, whereas the 


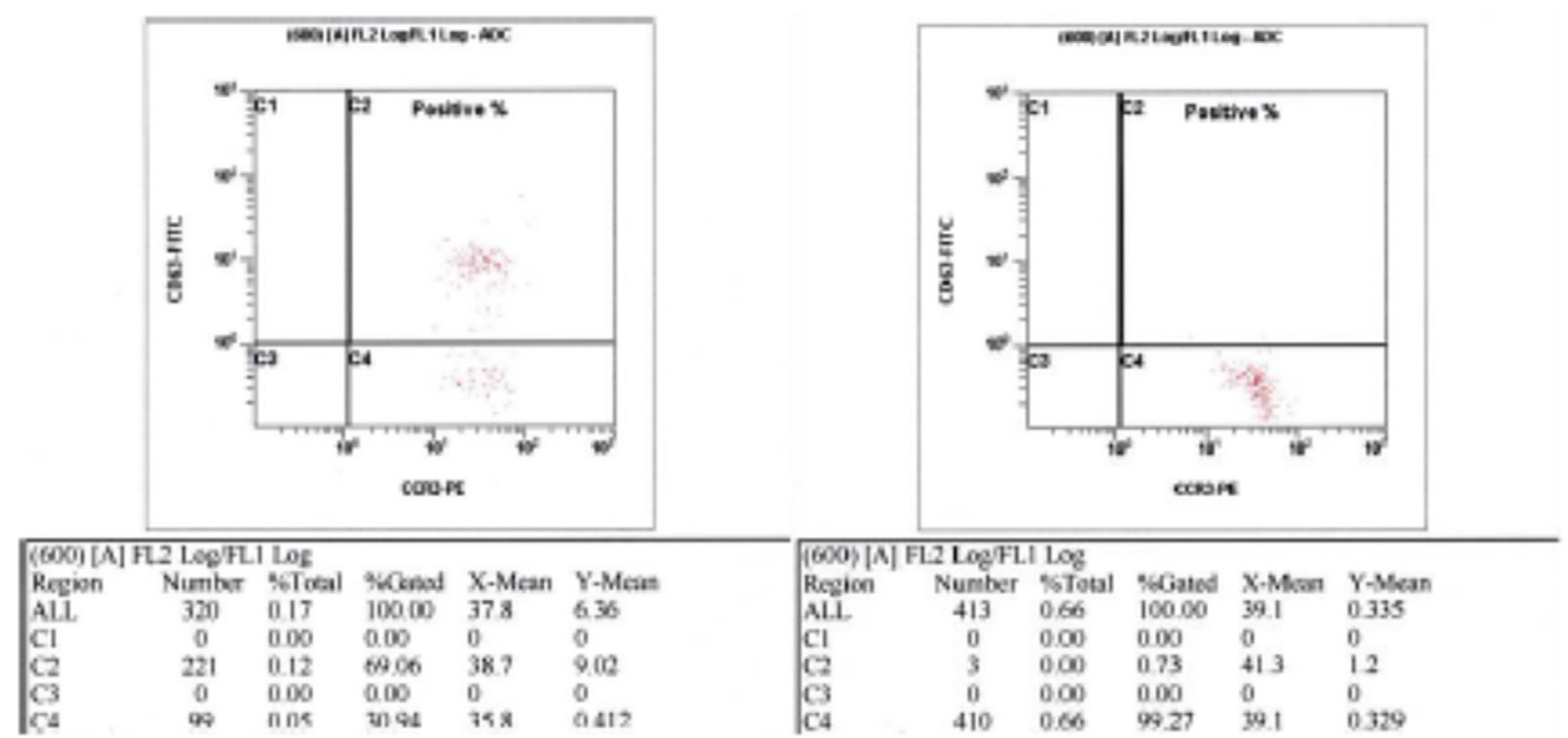

184

185

186

187

188

189

190

191

192

193

194

195

196

197

198

199

200

201

202

203

204

205

206

207

208

209

210

211

212

213

214

215

216

217

218

219

220

FIGURE 2. Basophil activation test results: diphenhydramine (top left); negative control without allergen (top right); loratadine (bottom \& $5^{5}$ right); and chlorphenamine (bottom left). A total of $69 \%$ of gated basophils (CCR3+ cells) showed upregulation of CD63 when stimulated with diphenhydramine $(5 \mu \mathrm{g} / \mathrm{mL})$, compared with only $0.73 \%$ in the negative control plot; $1.55 \%$ and $0.96 \%$ of gated basophils showed upregulation of CD63 when stimulated with loratadine $(50 \mu \mathrm{g} / \mathrm{mL})$ and chlorphenamine $(4 \mu \mathrm{g} / \mathrm{mL})$, respectively.

other demonstrated intradermal reactivity to diphenhydramine; our patient showed a positive skin prick test result. It is interesting to note the positive skin reactivity from an antihistamine epicutaneous prick. Histamine is an important but not the only mediator involved in the induction of wheal and flare response. Other mediators also contribute, such as platelet-derived growth factors, leukotrienes, and prostaglandins. Hence, it is likely that the skin reaction still occurred after degranulation of cutaneous mast cells with the antihistamine, diphenhydramine, as a stimulant in this unusual case. Although details of any potential excipient of the nasal drops and the oral formulation used during the anaphylaxis episodes were not available, the antihistamines we used for skin and BATs were pure without mixtures with preservatives. Therefore, the patient most likely has hypersensitivity to diphenhydramine.

BAT, a flow-based assay, is increasingly used in drug allergy workup. ${ }^{4,6-9}$ In general, the sensitivity of BAT is higher than serum-specific IgE testing, but results can vary depending on the different drug items under investigation. ${ }^{9}$ Drug preparation and optimal dose for stimulation are the most crucial steps for BAT. Because there is no previously published protocol in the literature, we performed the assay with serial concentrations of 5,50 , 500 , and $5000 \mu \mathrm{g} / \mathrm{mL}$ of diphenhydramine, and positive results were noted at $5 \mu \mathrm{g} / \mathrm{mL}$, as well as 50 and $500 \mu \mathrm{g} / \mathrm{mL}$. 
The chemical structure of antihistamines is quite different between groups that are unlikely to share cross-reactivity. Indeed, including the previously reported cases, all 3 patients with diphenhydramine allergy were successfully challenged with an alternative antihistamine. In this case, cetirizine showed a positive result in BAT. It would be interesting to test whether positivity in BAT correlates with cetirizine intolerance. However, the patient declined cetirizine challenge after he tolerated loratadine.

Although severe allergic reactions to a drug commonly used for allergy treatment are rare, one should remain vigilant of this possibility. Skin testing and BATs showed some promise for confirming the diagnosis of diphenhydramine allergy and identifying an alternative, although more research will be needed to validate the methodology. Moreover, because neither skin test nor BAT is an absolute proof of an IgE-mediated process, one has to interpret investigation findings along with clinical presentation carefully.

Q1 ${ }^{\mathrm{a} D e p a r t m e n t}$ of Pathology, Queen Mary Hospital, Hong Kong

${ }^{b}$ Department of Medicine, Queen Mary Hospital, Li Ka Shing Faculty of Medicine, The University of Hong Kong, Hong Kong

${ }^{c}$ Department of Paediatrics and Adolescent Medicine, Queen Mary Hospital, Li Ka Shing Faculty of Medicine, The University of Hong Kong, Hong Kong

Q2 No funding was received for this work

Conflicts of interest: The authors declare that they have no relevant conflicts of interest.

Received for publication September 12, 2017; revised October 10, 2017; accepted for publication October 24, 2017.

Available online
Corresponding author: Elaine Y.L. Au, MBBS, FHKAM (Medicine), FRCPA, FHKAM (Pathology), D (ABMLI), Division of Clinical Immunology, Department of Pathology, Room 511, Clinical Pathology Building, Queen Mary Hospital, 102 Pokfulam Road, Hong Kong. E-mail: elaineauyl@gmail.com.

2213-2198

(C) 2017 American Academy of Allergy, Asthma \& Immunology

https://doi.org/10.1016/j.jaip.2017.10.032

\section{REFERENCES}

1. Mur Gimeno P, Alfaya Arias T, Iglesias Aranzazu M, Lombardero Vega M, Sastre B. Anaphylactic shock caused by antihistamines. J Investig Allergol Clin Immunol 2011;21:321-2.

2. Barranco P, Lopez-Serrano MC, Moreno-Ancillo A. Anaphylactic reaction due to diphenhydramine. Allergy 1998;53:814.

3. Schwartz LB, Metcalfe DD, Miller JS, Earl H, Sullivan T. Tryptase levels as an indicator of mast-cell activation in systemic anaphylaxis and mastocytosis. N Engl J Med 1987;316:1622-6.

4. Decuyper II, Mangodt EA, Van Gasse AL, Claesen K, Uyttebroek A, Faber M, et al. In vitro diagnosis of immediate drug hypersensitivity anno 2017: potentials and limitations. Drugs R D 2017;17:265-78.

5. Chisholm DG, Calder I, Peterson D, Powell M, Moult P. Intranasal chlorhexidine resulting in anaphylactic circulatory arrest. BMJ 1997;315:785.

6. Sanz ML, Gamboa PM, Antepara I, Uasuf C, Vila L, Garcia-Aviles C, et al. Flow cytometric basophil activation test by detection of CD63 expression in patients with immediate-type reactions to beta lactam antibiotics. Clin Exp Allergy 2002; 32:277-86.

7. Mangodt EA, Van Gasse AL, Bastiaensen A, Decuyper II, Uyttebroek A, Faber M, et al. Flow-assisted basophil activation tests in immediate drug hypersensitivity: two decades of Antwerp experience. Acta Clin Belg 2016;71:19-25.

8. Ebo DG, Sainte-Laudy J, Bridts $\mathrm{CH}$, Mertens $\mathrm{CH}$, Hagendorens MM, Schuerwegh AJ, et al. Flow-assisted allergy diagnosis: current applications and future perspectives. Allergy 2006;61:1028-39.

9. Hamilton RG, Franklin Adkinson N Jr. In vitro assays for the diagnosis of IgEmediated disorders. J Allergy Clin Immunol 2004;114:213-25. 